\title{
Nutritional correlates of monetary diet cost in young, middle-aged and older Japanese women
}

\author{
Keiko Shiraki ${ }^{1}$, Kentaro Murakami ${ }^{2}$, Hitomi Okubo ${ }^{3}$, M. Barbara E. Livingstone ${ }^{4}$, Satomi Kobayashi ${ }^{2}$, \\ Hitomi Suga $^{5}$, Satoshi Sasaki ${ }^{2}$ and The Three-Generation Study of Women on Diets and Health \\ Study Group \\ ${ }^{1}$ Department of Nutrition, School of Human Cultures, University of Shiga Prefecture, Shiga, Japan \\ ${ }^{2}$ Department of Social and Preventive Epidemiology, School of Public Health, University of Tokyo, Tokyo, Japan \\ ${ }^{3}$ Department of Health Promotion, National Institute of Public Health, Saitama, Japan \\ ${ }^{4}$ Northern Ireland Centre for Food and Health, Ulster University, Coleraine, UK \\ ${ }^{5}$ Department of Social and Preventive Epidemiology, Graduate School of Medicine, University of Tokyo, Tokyo, Japan
}

(Received 9 September 2016 - Final revision received 21 February 2017 - Accepted 31 March 2017)

Journal of Nutritional Science (2017), vol. 6, e22, page 1 of 11

doi:10.1017/jns.2017.18

Abstract

Studies in many Western countries have consistently shown that monetary diet cost is positively associated with diet quality, but this may not necessarily be the case in Japan. This cross-sectional study examined the nutritional correlates of monetary diet cost among 3963 young (all 18 years old), 3800 middle-aged (mean age 48 years) and 2211 older (mean age 74 years) Japanese women. Dietary intakes were assessed using a comprehensive self-administered diet history questionnaire for young and middle-aged women and a brief self-administered diet history questionnaire for older women. Monetary diet cost was estimated using retail food prices. Total vegetables, fish and shellfish, green and black tea, white rice, meat, fruit and alcoholic beverages contributed most (79-89 \%) to interindividual variation in monetary diet cost. Multiple regression analyses showed that monetary diet cost was negatively associated with carbohydrate intake, but positively with intakes of all other nutrients examined (including not only dietary fibre and key vitamins and minerals but also saturated fat and $\mathrm{Na}$ ) in all generations. For food group intakes, irrespective of age, monetary diet cost was associated inversely with white rice and bread but positively with pulses, potatoes, fruit, total vegetables, fruit and vegetable juice, green and black tea, fish and shellfish, and meat. In conclusion, in all three generations of Japanese women and contrary to Western populations, monetary diet cost was positively associated with not only healthy dietary components (including fruits, vegetables, fish and shellfish, dietary fibre, and key vitamins and minerals), but also less healthy components (including saturated fat and $\mathrm{Na}$ ).

Key words: Diet: Monetary cost: Women: Japan

Although food choice is influenced by many factors including taste, convenience, concerns about nutrition and body weight, the price of food is clearly an important determinant ${ }^{(1,2)}$. In general, foods that are energy-dense but nutrient-poor such as fat and oils, and sugar and confectioneries, provide dietary energy at lowest cost, whereas the price of foods that are nutrient-dense such as fish and shellfish, vegetables and fruits is much higher ${ }^{(3-7)}$.
Previous studies from the $\mathrm{UK}^{(4,8,9)}$, Spain ${ }^{(3,10)}$, France ${ }^{(6,11,12)}$, the Netherlands ${ }^{(13)}$ and the USA ${ }^{(5,14-18)}$ all suggest that healthier diets cost more. Typically, higher-quality diets are characterised by higher intakes of whole grains, lean meats, fruits and vegetables, and by lower intakes of fats and sugars, and refined grains ${ }^{(19,20)}$. Further, the positive association between monetary diet cost and diet quality may explain the socio-economic

Abbreviations: BDHQ, brief diet history questionnaire; DHQ, diet history questionnaire; EI, energy intake; PAL, physical activity level.

* Corresponding author: Dr K. Murakami, fax +8135841 7873, email kenmrkm@m.u-tokyo.ac.jp

(C) The Author(s) 2017. This is an Open Access article, distributed under the terms of the Creative Commons Attribution licence (http://creative commons.org/licenses/by/4.0/), which permits unrestricted reuse, distribution, and reproduction in any medium, provided the original work is properly cited. 
gradient in health widely observed in Western countries ${ }^{(7,8,20-22)}$, with higher socio-economic status associated with better health ${ }^{(23,24)}$.

However, in Japan, where unclear or inverse associations between socio-economic status and health status are observed $^{(25,26)}$, the association between monetary diet cost and diet quality may be more complex than that observed in Western countries. Indeed, in selected Japanese population groups, monetary diet cost was associated not only with healthier (including higher intakes of dietary fibre, key vitamins and minerals, fruits, vegetables and lower dietary energy density) but also less healthy diets (including higher intakes of saturated fat and $\mathrm{Na})^{(27-29)}$.

Further investigation in a general Japanese population is clearly needed because the relationship between monetary diet cost and diet quality is an important issue. Therefore, the aim of the present cross-sectional study was to examine nutritional correlates of monetary diet cost with food and nutrient intake in young, middle-aged and older Japanese women. Specifically, we investigated the food contributions to diet cost, the inter-individual variation in diet cost explained by each food group, and the association between diet cost and nutrient and food group intake after adjustment for a range of potential confounding factors.

\section{Subjects and methods}

\section{Survey design}

The present cross-sectional study was based on data from the Three-Generation Study of Women on Diets and Health, a questionnaire survey conducted in northern and western Japan in 2011 and in eastern Japan in 2012. The study design and survey procedure have been described in detail ${ }^{(30)}$. Briefly, two questionnaires on dietary habits and lifestyle factors were distributed to a total of 7016 dietetic students from eighty-five higher education institutions in thirty-five of forty-seven prefectures in Japan during the orientation session or the first lecture designed for freshmen in April 2011 or 2012. Each student was also requested to invite his/her mother and grandmother to join the study and to distribute similar questionnaires to those agreeing to take part. Recruitment priority for the grandmother generation was given to the maternal or, if unavailable, paternal grandmother, followed by his/her female acquaintance aged 65-89 years. In total, 4933 students, including 4656 women and 277 men (response rate $70.3 \%$ ), 4044 mothers $(57.6 \%)$, and 2332 women from the grandmothers' generation $(33.2 \%)$ answered both questionnaires.

This study was conducted according to the guidelines laid down in the Declaration of Helsinki and all procedures involving human subjects were approved by the Ethics Committee of the University of Tokyo Faculty of Medicine. Written informed consent was obtained from each subject and also from a parent/guardian for subjects aged $<20$ years.

\section{Analytic sample}

In the present study, we considered that the students (excluding males), mothers and grandmothers (including acquaintances) consisted of groups of young, middle-aged and older women, respectively.

For the youngest group, we selected female students aged 18 years ( $n$ 4065). We then excluded those not only living in eastern Japan but who also participated in the 2011 survey (because it was assumed that they could not report their usual dietary habits and lifestyle due to the occurrence of the Great East Japan Earthquake in March 2011) (n 39), those who answered the questionnaires after 19 May to minimise the influence of dietetic education ( $n$ 56) and those whose data were derived from the institution where the response rate was extremely low $(2 \%)\left(\begin{array}{ll}n & 2\end{array}\right)$. We further excluded those with missing information on the variables of interest ( $n$ 5).

For the analysis of middle-aged women, we selected mothers aged 34 to 60 years ( $n$ 4012). We then excluded those not only living in eastern Japan but who also participated in the 2011 survey ( $n$ 63) and those whose data were derived from the institution with the low response rate $(n 2)$. We further excluded those with erroneous or missing information on the variables of interest ( $n$ 147).

For the analysis of older women, we selected grandmothers (and acquaintances) aged 61 to 94 years ( $n$ 2325). We then excluded those not only living in eastern Japan but who also participated in the 2011 survey ( $n$ 47) and those whose data were derived from the institution with the low response rate (n 1). We further excluded those with erroneous or missing information on the variables of interest ( $n$ 66). Consequently, the final sample sizes were 3963, 3800 and 2211 for young, middle-aged and older women, respectively.

\section{Dietary assessment}

Dietary habits during the preceding month were assessed using a comprehensive diet history questionnaire (DHQ) ${ }^{(31-33)}$ for young and middle-aged women and a brief diet history questionnaire $(\mathrm{BDHQ})^{(31,32)}$ for older women. Responses to the DHQ and BDHQ, as well as to the lifestyle questionnaire, were checked at least once by survey staff at the study centre. If any missing or erroneous responses were given to questions which were considered essential for analysis, the participant was asked to complete these again. Details of the structure and calculation method of dietary intake of the DHQ and BDHQ have been published elsewhere ${ }^{(31-33)}$. Briefly, the DHQ and BDHQ are structured, self-administered questionnaires that ask about the consumption frequency (and portion size in DHQ) of selected foods commonly consumed in Japan, as well as general dietary behaviour and usual cooking methods ${ }^{(33,34)}$. Estimates of the daily intake for foods (151 items in DHQ and fifty-eight items in BDHQ), energy and selected nutrients were calculated using an ad hoc computer algorithm for the DHQ and BDHQ, which was based on the Standard Tables of Food Composition in Japan ${ }^{(35)}$. To minimise the influence of dietary misreporting ${ }^{(36,37)}$, energy-adjusted values of dietary variables were calculated based on the density method (i.e. percentage of energy for energy-providing nutrients and amount per $4184 \mathrm{~kJ}$ of energy for other nutrients and foods). A relative validity of the DHQ 
and BDHQ has been previously investigated among ninetytwo women aged 31-69 years using a 16-d weighed dietary record as reference ${ }^{(31,32)}$. Briefly, for the DHQ, the median value of Spearman's correlation coefficients for food groups was 0.43 (range -0.09 to 0.77 ), and that of Pearson's correlation coefficients for nutrients was 0.57 (range 0.27 to 0.87 ). The corresponding values for the BDHQ were 0.44 (range 0.14 to 0.82 ) and 0.54 (range 0.34 to 0.87 ), respectively.

\section{Calculation of monetary diet cost}

Monetary diet cost (Japanese yen/d) was calculated by multiplying the amount of each food item consumed (derived from the DHQ $(\mathrm{g} / \mathrm{d}$ ) for young and middle-aged women or the BDHQ $(\mathrm{g} / \mathrm{d})$ for older women) by estimated price of food (Japanese yen $/ \mathrm{g}$ ) and summing the products. The procedure for estimating costs was based on the assumption that all foods were purchased and then prepared and consumed at home, with some exceptions (French fries and doughnuts) described below. Sale prices were not used to determine costs. Costs of composite foods such as pizza were calculated using prices of frozen equivalents. Calculation included correction for preparation and waste.

The price of food items was obtained from two sources. The first was the National Retail Price Survey of $2004^{(38)}$. This survey was conducted in 167 villages, towns and cities, and average prices were calculated as mean value of all survey areas, weighted for population size. The second source was information on price from the websites of supermarkets (Seiyu, Japan) and fast-food restaurants (McDonalds, Japan and Mister Donut, Japan) located throughout Japan. For French fries and doughnuts, the price was determined by that from fast-food restaurants, as these items are usually purchased in such restaurants in Japan.

To determine the price of individual food items, we used the following three procedures:

(1) Direct matching. Each food in the DHQ and BDHQ was directly matched to foods appearing in the National Retail Price Survey. This procedure was used to determine the price of 179 of the 236 items in the DHQ (76\%) and 121 of the 150 in the BDHQ (81\%).

(2) Comparable food items. When exact matching to a food appearing in the National Retail Price Survey was not possible, the closest alternative was chosen. This procedure was used to determine the price of forty-two items in the DHQ (18\%) and twenty-seven items in the BDHQ $(18 \%)$.

(3) Website. When no comparable food in the National Retail Price Survey was available, prices were taken from the websites. This procedure was used to determine the price of fifteen items in the DHQ (6\%) and two in the BDHQ (1\%).

The monetary cost (Japanese yen/100 g) of each food item (as well as the percentage contribution to total cost in the present populations) is listed in Supplementary Table S1 for DHQ and Supplementary Table S2 for BDHQ. As crude monetary diet cost was highly correlated with energy intake (EI) in the present populations (Pearson correlation coefficient 0.81 to 0.90 ), energy-adjusted monetary diet cost by the density method (Japanese yen/4184 kJ) was used in the present study. A detailed description of the cost calculation methods has been published elsewhere ${ }^{(27,39)}$. In a group of ninety-two Japanese women aged 31-69 years, Pearson's correlation coefficient between DHQ and 16-d weighed dietary records was $0.60^{(39)}$, and the correlation for BDHQ was 0.44 (S Sasaki and K Murakami, unpublished results), suggesting satisfactory reliability of the DHQ and BDHQ in terms of monetary diet cost.

The food price database was created using data collected in 2004, whereas the present data were collected in 2011 and 2012. Despite this, a correction for inflation was not considered necessary since it is the relative, not absolute, costs within the population that are of interest for the purposes of the present study ${ }^{(8)}$.

\section{Assessment of other variables}

All the variables used were based on each participant's selfreported information. Age at the time of the survey was calculated based on birth date. Residential area was grouped into six regions (Hokkaido and Tohoku, Kanto, Hokuriku and Tokai, Kinki, Chugoku and Shikoku, or Kyushu) and also into three categories according to population size (city with a population $\geq 1$ million, city with a population $<1$ million, or town and village). Living status (not considered in middle-aged women because almost all lived with their family) was grouped into three categories (living alone, living with family, or living with others), but for older women those living with others were added to those living with family because of the very small number of subjects ( $n$ 4). BMI was calculated as body weight $(\mathrm{kg})$ divided by square of body height $(\mathrm{m})$. Weight status was grouped into three categories: underweight (BMI $<18.5 \mathrm{~kg} / \mathrm{m}^{2}$ ), normal weight (BMI $\geq 18.5$ to $<25 \mathrm{~kg} / \mathrm{m}^{2}$ ) and overweight $\left(B M I \geq 25 \cdot 0 \mathrm{~kg} / \mathrm{m}^{2}\right)^{(40)}$. All the results did not change when using the Asian BMI cut-offs recommended by the $\mathrm{WHO}^{(41)}$ (data not shown). Information on current smoking (yes or no), current alcohol drinking (yes or no), dietary supplement use (not only vitamins/minerals but also specialty supplements such as botanicals, other herbs, etc.; yes or no) and prescription medicine use (yes or no) was also used. Eating out was categorised as $\leq 3$ times/month, once per week, 2-3 times/week, or $\geq 4$ times/week (not available in older women). Physical activity was computed as the average total metabolic equivalent-hours score per $\mathrm{d}$ on the basis of the frequency and duration of seven activities (walking, bicycling, standing, running, high-intensity activities, sleeping, and sedentary activity) over the preceding months ${ }^{(42)}$, which was categorised into quartiles. For only middle-aged women, occupation was considered (housewife, part-time job, or full-time job). Except for young women, education level was categorised as low, middle, and high ( $\leq 12$ years, $13-15$ years, and $\geq 16$ years for middle-aged women and $\leq 9$ years, 10-12 years, and $\geq 13$ years for older women, respectively). Current marital status (yes or no) was also considered for middle-aged and older women.

Misreporting of EI was evaluated on the basis of the ratio of EI:BMR (the Goldberg cut-off) ${ }^{(43)}$. Subjects were identified as 
plausible, under- and over-reporters of EI according to whether the individual's ratio was within, below or above the $95 \%$ confidence limits for agreement between EI:BMR and the respective physical activity level (PAL). In the present analysis, the PAL for sedentary lifestyle (i.e. 1.55) ${ }^{(43)}$ was applied for all subjects. This decision was not only because of a lack of a validated measure of physical activity but also because in all generations, time spent on sedentary activity (range of mean: 11.65 to $14.00 \mathrm{~h} / \mathrm{d}$ ) was predominant compared with other activities: walking (1.31 to $1.95 \mathrm{~h} / \mathrm{d})$, bicycling $(0.14$ to $0.31 \mathrm{~h} / \mathrm{d})$, standing $(1.64$ to $2.99 \mathrm{~h} / \mathrm{d})$, running $(0.02$ to $0.04 \mathrm{~h} / \mathrm{d})$, high-intensity activities $(0.05$ to $0.06 \mathrm{~h} / \mathrm{d})$ and sleeping $(6.22$ to $7.97 \mathrm{~h} / \mathrm{d})$. BMR was estimated according to an equation specifically developed for Japanese women, as follows: BMR $(\mathrm{kJ} / \mathrm{d})=(0.0481 \times$ body weight $(\mathrm{kg})+0.0234 \times$ body height $(\mathrm{cm})-0.0138 \times$ age (years) -0.9708$)^{(44,45)}$. The $95 \%$ confidence limits for agreement (upper and lower cut-off values) between EI:BMR and the PAL were calculated, taking into account CV in intakes and other components of energy balance (i.e. the within-subject variation in EI: $23 \%$; the precision of the estimated BMR relative to the measured BMR: $8.5 \%$; and the between-subject variation in PAL: $15 \%)^{(43)}$. Consequently, under-, plausible and overreporters were defined as having EI:BMR $<1 \cdot 09,1 \cdot 09-2 \cdot 21$ and $>2 \cdot 21$, respectively ${ }^{(43)}$.

\section{Statistical analysis}

All statistical analyses were performed for young, middle-aged and older women separately, using SAS statistical software version 9.3 (SAS Institute Inc.). All reported $P$ values are twotailed, and $P<0.05$ was considered statistically significant. Descriptive data are presented as means and standard deviations for continuous variables, except for food group intakes for which medians and 25th and 75th percentiles are used, and numbers and percentages of subjects for categorical variables. Differences in monetary diet cost across categories of selected characteristics were examined by ANOVA.

Using the PROC REG procedure, stepwise forward regression analyses were carried out to investigate the contribution of the selected twenty food groups for young and middle-aged women and eighteen food groups for older women to the inter-individual variation in monetary diet cost. The significance level for entry into the model was set at $P<0 \cdot 1$. For those food groups contributing at least $1 \%$ variation, multiple regression analyses were performed (using the PROC REG procedure) with predictive food groups as explanatory variables and monetary diet cost as the response variable.

Multiple regression analyses were performed to explore the association of monetary diet cost (the explanatory variable) with food group and nutrient intakes (response variables). Using the PROC REG procedure, we calculated the adjusted regression coefficients (with standard errors) of variation of intakes of selected food groups and nutrients by a 100 Japanese yen increase in monetary diet cost (per $4184 \mathrm{~kJ}$ ). Potential confounding factors considered included survey year, residential block, size of residential area, weight status, current smoking, current alcohol drinking, dietary supplement use, medication use, physical activity and dietary reporting status. Further adjustment was made for living status and eating out in young women; eating out, occupation, education, current marital status and age ( $<45,45-49$, or $\geq 50$ years) in middle-aged women; and living status, education, current marital status and age (61-69, 70-74, 75-79, or $\geq 80$ years) in older women. These analysis were repeated after excluding under- and over-reporters. Monetary diet cost was analysed continuously after confirming the linearity of relationships using quintile categories.

\section{Results}

\section{Population characteristics}

All of the young women were 18 years old, whereas mean age was 47.7 (SD 3.9) years (range 34-60 years) in middle-aged women and 74.4 (SD 5.2) years (range 61-94 years) in older women (Table 1). Mean EI:BMR was 1.47 (SD 0.50) in young women, $1.66(0.49)$ in middle-aged women, and 1.86 (SD 0.61) in older women. Mean value of energy-adjusted monetary diet cost was 479 (SD 98) Japanese yen/4184 kJ for young women, 531 (SD 101) Japanese yen/4184 kJ for middle-aged women, and 629 (SD 105) Japanese yen/4184 kJ for older women.

Percentage contribution of each food group to monetary diet cost

The highest contributor to monetary diet cost differed by generations: meat $(17.2 \%)$ in young women, total vegetables $(15.9 \%)$ in middle-aged women, and fish and shellfish $(22.4 \%)$ in older women (Table 2). However, in all generations, the top four contributors were the same: meat, total vegetables, sugar and confectioneries, and fish and shellfish (54.8 to $60.3 \%$ in total). Other important contributors included white rice (5.8 to $7.3 \%$ ) and green and black tea $(5.0$ to $7.7 \%)$ in all generations, alcoholic beverages $(4.8 \%)$ in middle-aged women, and fruit $(6.7 \%)$ in older women.

\section{Contribution of food groups to inter-individual variation in monetary diet cost}

Total vegetables, fish and shellfish, green and black tea, white rice (only young and older women), meat, fruit and alcoholic beverages (only middle-aged and older women) contributed most $(79$ to $89 \%$ ) to inter-individual variation in monetary diet cost (Table 3). Other contributors included soft drinks $(3 \%)$, sugar and confectioneries (3\%), and fruit and vegetable juice $(1 \%)$ in young women, soft drinks $(3 \%)$, sugar and confectioneries $(3 \%)$, and coffee $(4 \%)$ in middle-aged women, and sugar and confectioneries $(1 \%)$ in older women.

\section{Associations between monetary diet cost and selected characteristics}

Monetary diet cost differed significantly according to many of the selected characteristics examined (Supplementary Table S3). For residential block, monetary diet cost was the highest in Kinki and the lowest in Hokkaido and Tohoku 
Table 1. Basic and dietary characteristics of young, middle-aged and older Japanese women (Arithmetic mean values and standard deviations or median values and 25 th and 75 th percentiles)

\begin{tabular}{|c|c|c|c|c|c|c|}
\hline & \multicolumn{2}{|c|}{ Young ( $n$ 3963) } & \multicolumn{2}{|c|}{ Middle-aged ( $n 3800)$} & \multicolumn{2}{|c|}{ Older ( $n$ 2211) } \\
\hline & $\begin{array}{c}\text { Arithmetic } \\
\text { mean }\end{array}$ & SD & $\begin{array}{c}\text { Arithmetic } \\
\text { mean }\end{array}$ & SD & $\begin{array}{c}\text { Arithmetic } \\
\text { mean }\end{array}$ & SD \\
\hline Age (years) & 18 & 0 & $47 \cdot 7$ & 3.9 & 74.4 & $5 \cdot 2$ \\
\hline Body height $(\mathrm{cm})$ & $157 \cdot 7$ & $5 \cdot 3$ & $157 \cdot 3$ & $5 \cdot 1$ & $150 \cdot 4$ & 5.5 \\
\hline Body weight (kg) & 52.0 & 7.8 & 54.5 & 8.2 & $51 \cdot 6$ & 7.9 \\
\hline $\mathrm{BMI}\left(\mathrm{kg} / \mathrm{m}^{2}\right)$ & $20 \cdot 9$ & $2 \cdot 8$ & $22 \cdot 0$ & 3.1 & $22 \cdot 8$ & 3.2 \\
\hline $\mathrm{El}(\mathrm{kJ} / \mathrm{d})$ & 7242 & 2399 & 7717 & 2231 & 7369 & 2277 \\
\hline El:BMR & 1.47 & 0.50 & 1.66 & 0.49 & 1.86 & 0.61 \\
\hline \multicolumn{7}{|l|}{ Monetary diet cost } \\
\hline Crude diet cost (Japanese yen/d)* & 838 & 351 & 981 & 339 & 1115 & 417 \\
\hline Energy-adjusted diet cost (Japanese yen/4184 kJ)* & 479 & 98 & 531 & 101 & 629 & 105 \\
\hline \multicolumn{7}{|l|}{ Nutrient intake } \\
\hline Protein (\% of energy) & 13.0 & $2 \cdot 2$ & 13.7 & $2 \cdot 0$ & $16 \cdot 9$ & $3 \cdot 2$ \\
\hline Total fat (\% of energy) & 29.4 & $6 \cdot 3$ & $29 \cdot 1$ & 5.9 & $25 \cdot 7$ & $5 \cdot 1$ \\
\hline Saturated fat (\% of energy) & $8 \cdot 3$ & $2 \cdot 3$ & 8.0 & $2 \cdot 0$ & $6 \cdot 7$ & 1.6 \\
\hline Monounsaturated fat (\% of energy) & $10 \cdot 4$ & $2 \cdot 7$ & $10 \cdot 4$ & 2.5 & 8.9 & $2 \cdot 0$ \\
\hline Polyunsaturated fat (\% of energy) & $6 \cdot 5$ & 1.6 & $6 \cdot 7$ & 1.5 & $6 \cdot 4$ & 1.4 \\
\hline Carbohydrate (\% of energy) & $56 \cdot 3$ & $7 \cdot 3$ & $54 \cdot 2$ & $7 \cdot 1$ & 55.9 & 7.4 \\
\hline Alcohol (\% of energy) & 0.1 & 0.7 & 1.8 & 4.1 & 0.5 & $2 \cdot 3$ \\
\hline Dietary fibre (g/4184 kJ) & $6 \cdot 2$ & $2 \cdot 0$ & $6 \cdot 7$ & $2 \cdot 0$ & $8 \cdot 0$ & $2 \cdot 2$ \\
\hline Cholesterol (mg/4184 kJ) & 171 & 75 & 165 & 57 & 231 & 76 \\
\hline $\mathrm{Na}(\mathrm{mg} / 4184 \mathrm{~kJ})$ & 2134 & 639 & 2279 & 618 & 2558 & 526 \\
\hline K (mg/4184 kJ) & 1030 & 280 & 1194 & 276 & 1631 & 399 \\
\hline Ca (mg/4184 kJ) & 246 & 94 & 267 & 93 & 367 & 112 \\
\hline $\mathrm{Mg}(\mathrm{mg} / 4184 \mathrm{~kJ})$ & 113 & 28 & 131 & 27 & 159 & 33 \\
\hline $\mathrm{Fe}(\mathrm{mg} / 4184 \mathrm{~kJ})$ & 3.6 & 0.9 & 3.7 & 0.9 & $5 \cdot 1$ & $1 \cdot 2$ \\
\hline Vitamin A $(\mu \mathrm{g} / 4184 \mathrm{~kJ}) \dagger$ & 271 & 206 & 288 & 185 & 476 & 258 \\
\hline Vitamin E (mg/4184 kJ)‡ & $4 \cdot 1$ & $1 \cdot 2$ & $4 \cdot 2$ & $1 \cdot 1$ & 4.5 & 1.0 \\
\hline Folate $(\mu \mathrm{g} / 4184 \mathrm{~kJ})$ & 147 & 52 & 156 & 48 & 239 & 75 \\
\hline Vitamin C (mg/4184 kJ) & $46 \cdot 5$ & 23.5 & $47 \cdot 9$ & 23.2 & 85.5 & $31 \cdot 2$ \\
\hline \multicolumn{7}{|l|}{ Food intake $(\mathrm{g} / 4184 \mathrm{~kJ}) \S$} \\
\hline White rice & $148 \cdot 3$ & $98.8,201.5$ & $132 \cdot 1$ & $87.9,178.4$ & 159.9 & $116 \cdot 6,210 \cdot 4$ \\
\hline Other grains $\| \emptyset$ & 0 & 0,0 & 0 & 0,0 & - & - \\
\hline Noodles & $28 \cdot 3$ & $13 \cdot 1,49 \cdot 2$ & $27 \cdot 9$ & $13 \cdot 5,46 \cdot 4$ & $25 \cdot 0$ & $13 \cdot 9,41 \cdot 1$ \\
\hline Bread & 14.6 & $6 \cdot 2,25 \cdot 8$ & $15 \cdot 4$ & $7 \cdot 0,27 \cdot 1$ & 17.4 & $6 \cdot 8,32 \cdot 9$ \\
\hline Other grain products $\eta^{\star *}$ & $4 \cdot 3$ & $0,10.4$ & 3.5 & $0,9.9$ & - & - \\
\hline Pulses & 19.6 & $11 \cdot 3,34.4$ & $27 \cdot 9$ & $17 \cdot 5,43 \cdot 1$ & $42 \cdot 9$ & $28 \cdot 5,61 \cdot 6$ \\
\hline Potatoes & $12 \cdot 5$ & $8 \cdot 1,19 \cdot 7$ & $12 \cdot 9$ & $8 \cdot 5,21 \cdot 3$ & $28 \cdot 1$ & $14 \cdot 7,45 \cdot 3$ \\
\hline Sugar and confectioneries & $49 \cdot 6$ & $35 \cdot 3,67 \cdot 8$ & 44.6 & $31 \cdot 6,60 \cdot 1$ & 37.5 & $21 \cdot 4,56 \cdot 1$ \\
\hline Fat and oil & 11.9 & $8 \cdot 7,16 \cdot 1$ & $11 \cdot 7$ & $8 \cdot 8,15 \cdot 0$ & 8.2 & $5 \cdot 6,10 \cdot 9$ \\
\hline Fruit & $16 \cdot 0$ & $8 \cdot 0,31 \cdot 0$ & $21 \cdot 8$ & $10 \cdot 1,44 \cdot 0$ & $55 \cdot 2$ & $29 \cdot 0,87.4$ \\
\hline Total vegetables & 97.4 & $64 \cdot 1,139.9$ & 114.2 & $81 \cdot 8,155 \cdot 7$ & $168 \cdot 2$ & $126 \cdot 0,225 \cdot 1$ \\
\hline Alcoholic beverages & 0 & 0,0 & 0 & $0,33.1$ & 0 & 0,0 \\
\hline Fruit and vegetable juice & 3.6 & $0,32 \cdot 8$ & 0 & $0,15 \cdot 3$ & 0 & $0,18.8$ \\
\hline Green and black tea & $261 \cdot 2$ & $122 \cdot 4,444.9$ & 233.0 & $108 \cdot 2,382 \cdot 1$ & $256 \cdot 2$ & $141.4,349.9$ \\
\hline Coffee & 0 & $0,13 \cdot 1$ & 135.8 & $59 \cdot 0,239 \cdot 4$ & 58.0 & $4.5,110.1$ \\
\hline Soft drinks & 23.9 & $2 \cdot 2,65 \cdot 0$ & 7.5 & $0,35.4$ & 0.0 & $0,8 \cdot 1$ \\
\hline Fish and shellfish & $22 \cdot 8$ & $14 \cdot 3,32 \cdot 7$ & 28.0 & $19 \cdot 9,38 \cdot 3$ & 54.4 & $36 \cdot 0,75 \cdot 0$ \\
\hline Meat & 33.3 & $22 \cdot 8,46 \cdot 7$ & $34 \cdot 2$ & $23 \cdot 8,46 \cdot 7$ & 30.4 & $20 \cdot 0,41.5$ \\
\hline Eggs & $18 \cdot 2$ & $8 \cdot 3,30 \cdot 2$ & $16 \cdot 0$ & $9 \cdot 1,26 \cdot 5$ & $17 \cdot 7$ & $11 \cdot 4,28 \cdot 3$ \\
\hline Dairy products & $37 \cdot 0$ & $11 \cdot 8,85 \cdot 0$ & $54 \cdot 1$ & $18 \cdot 3,99 \cdot 8$ & $66 \cdot 3$ & $24.5,98.5$ \\
\hline
\end{tabular}

El, energy intake.

* 1 Japanese yen $=0.0099$ US dollars $=0.0088$ Euros $=0.0074$ British pounds (September 2016).

$\dagger$ Retinol equivalents.

$\ddagger \alpha$-Tocopherol.

$\S$ Median values and 25th and 75th percentiles

II Including white rice mixed with barley, white rice with germ, half-milled rice, $70 \%$ milled rice and brown rice.

१ Not available for older women because the brief-type diet history questionnaire used for this age group did not include these food groups.

${ }_{\star * *}$ Including pizza, Japanese-style pancakes and cornflakes.

for young women, but the highest in Kanto and the lowest in Hokuriku and Tokai for older women. For living status in young women, monetary diet cost was the highest for those living with family and the lowest for those living alone. Size of residential area and education were positively associated with monetary diet cost (except for young women). Among current smokers a higher mean value of monetary diet cost was observed only in middle-aged women. Current alcohol drinkers had a lower mean value of monetary diet cost in middle-aged women but a higher mean value in older 
Table 2. Percentage contribution of each food group to monetary diet cost in young, middle-aged and older Japanese women* (Ranks; arithmetic mean values and $95 \%$ confidence intervals)

\begin{tabular}{|c|c|c|c|c|c|c|c|c|c|}
\hline & \multicolumn{3}{|c|}{ Young (n 3963) } & \multicolumn{3}{|c|}{ Middle-aged ( $n$ 3800) } & \multicolumn{3}{|c|}{ Older ( $n$ 2211) } \\
\hline & Rank & Arithmetic mean & $95 \% \mathrm{Cl}$ & Rank & Arithmetic mean & $95 \% \mathrm{Cl}$ & Rank & Arithmetic mean & $95 \% \mathrm{Cl}$ \\
\hline Meat & 1 & $17 \cdot 2$ & $16 \cdot 9,17 \cdot 4$ & 2 & $15 \cdot 0$ & $14 \cdot 8,15 \cdot 2$ & 3 & $10 \cdot 7$ & $10.5,11.0$ \\
\hline Total vegetables & 2 & 14.8 & $14 \cdot 6,15 \cdot 1$ & 1 & $15 \cdot 9$ & $15 \cdot 7,16 \cdot 1$ & 2 & $16 \cdot 6$ & $16 \cdot 3,16 \cdot 8$ \\
\hline Sugar and confectioneries & 3 & $14 \cdot 2$ & $13 \cdot 9,14.4$ & 4 & $11 \cdot 3$ & $11 \cdot 1,11.5$ & 4 & $10 \cdot 6$ & $10 \cdot 3,10 \cdot 9$ \\
\hline Fish and shellfish & 4 & $11 \cdot 7$ & $11.5,11.9$ & 3 & $12 \cdot 7$ & $12 \cdot 5,12 \cdot 8$ & 1 & 22.4 & $22 \cdot 0,22 \cdot 8$ \\
\hline Green and black tea & 5 & $7 \cdot 7$ & $7.5,7.9$ & 5 & $6 \cdot 1$ & $6 \cdot 0,6 \cdot 3$ & 7 & $5 \cdot 0$ & $4 \cdot 8,5 \cdot 1$ \\
\hline White rice & 6 & $7 \cdot 3$ & $7 \cdot 1,7 \cdot 4$ & 6 & $5 \cdot 8$ & $5 \cdot 7,5 \cdot 9$ & 6 & $6 \cdot 0$ & $5 \cdot 8,6 \cdot 1$ \\
\hline Dairy products & 7 & $4 \cdot 2$ & $4 \cdot 0,4 \cdot 3$ & 8 & 4.7 & $4 \cdot 6,4 \cdot 8$ & 9 & $2 \cdot 3$ & $2 \cdot 2,2 \cdot 3$ \\
\hline Fruit & 8 & 3.5 & $3 \cdot 4,3 \cdot 6$ & 10 & 3.8 & $3.7,3.9$ & 5 & $6 \cdot 7$ & $6 \cdot 5,6 \cdot 8$ \\
\hline Pulses & 9 & $2 \cdot 8$ & $2 \cdot 7,2 \cdot 8$ & 11 & 3.3 & $3 \cdot 2,3 \cdot 4$ & 8 & $3 \cdot 1$ & $3 \cdot 0,3 \cdot 1$ \\
\hline Noodles & 10 & 2.5 & $2 \cdot 4,2 \cdot 6$ & 12 & $2 \cdot 2$ & $2 \cdot 1,2 \cdot 2$ & 13 & 1.6 & $1.5,1.6$ \\
\hline Soft drinks & 11 & 2.5 & $2 \cdot 3,2 \cdot 6$ & 14 & 1.6 & $1 \cdot 5,1 \cdot 7$ & 18 & 0.5 & $0.4,0.6$ \\
\hline Potatoes & 12 & 1.8 & $1.8,1.9$ & 15 & 1.5 & $1.5,1.6$ & 11 & $2 \cdot 2$ & $2 \cdot 1,2 \cdot 3$ \\
\hline Bread & 13 & $1 \cdot 8$ & $1 \cdot 7,1 \cdot 8$ & 13 & 1.6 & $1 \cdot 6,1.7$ & 10 & $2 \cdot 2$ & $2 \cdot 1,2 \cdot 3$ \\
\hline Fat and oil & 14 & 1.7 & $1 \cdot 6,1 \cdot 7$ & 16 & 1.4 & $1.3,1.4$ & 17 & 0.6 & $0.6,0.6$ \\
\hline Eggs & 15 & $1 \cdot 7$ & $1 \cdot 6,1 \cdot 7$ & 17 & $1 \cdot 3$ & $1 \cdot 3,1 \cdot 3$ & 14 & $1 \cdot 2$ & $1 \cdot 2,1 \cdot 2$ \\
\hline Fruit and vegetable juice & 16 & $1 \cdot 3$ & $1 \cdot 2,1 \cdot 4$ & 19 & 0.7 & $0.6,0.7$ & 16 & 0.8 & $0.7,0.9$ \\
\hline Other grain products $\ddagger$ & 17 & $1 \cdot 2$ & $1 \cdot 1,1 \cdot 2$ & 18 & 1.0 & $0.9,1.0$ & - & - & - \\
\hline Other grains $\ddagger \S$ & 18 & 0.7 & $0 \cdot 7,0.8$ & 20 & 0.6 & $0.6,0.7$ & - & - & - \\
\hline Coffee & 19 & 0.5 & $0.4,0.5$ & 9 & $4 \cdot 1$ & $4 \cdot 0,4 \cdot 2$ & 12 & 1.6 & $1 \cdot 5,1 \cdot 7$ \\
\hline Alcoholic beverages & 20 & 0.1 & $0.1,0.2$ & 7 & 4.8 & $4 \cdot 5,5 \cdot 1$ & 15 & 1.0 & $0.8,1 \cdot 2$ \\
\hline
\end{tabular}

${ }^{*}$ Food groups are listed in descending order of their arithmetic mean percentage contribution to monetary diet cost in young women. † Including pizza, Japanese-style pancakes and cornflakes.

$\ddagger$ Not available for older women because the brief-type diet history questionnaire used for this age group did not include these food groups.

$\S$ Including white rice mixed with barley, white rice with germ, half-milled rice, $70 \%$ milled rice and brown rice.

Table 3. Food groups contributing to the inter-individual variation in monetary diet cost (Japanese yen/4184 kJ) in young, middle-aged and older Japanese women*

(Regression coefficients with their standard errors and partial determination coefficients)

\begin{tabular}{|c|c|c|c|c|c|c|c|c|c|}
\hline & \multicolumn{3}{|c|}{ Young (n 3963) } & \multicolumn{3}{|c|}{ Middle-aged (n 3800) } & \multicolumn{3}{|c|}{ Older (n 2211) } \\
\hline & $\beta \dagger$ & SE† & Partial $R^{2}$ & $\beta \dagger$ & SE† & Partial $R^{2}$ & $\beta \dagger$ & SE† & Partial $R^{2}$ \\
\hline Model $R^{2}$ & & 0.88 & & & 0.89 & & & 0.90 & \\
\hline Total vegetables & 6.62 & 0.09 & 0.38 & 7.03 & 0.10 & 0.27 & $5 \cdot 30$ & 0.10 & 0.22 \\
\hline Fish and shellfish & 21.41 & 0.35 & 0.14 & $19 \cdot 84$ & 0.36 & 0.09 & $19 \cdot 86$ & 0.26 & 0.45 \\
\hline Green and black tea & 1.23 & 0.02 & 0.12 & $1 \cdot 14$ & 0.02 & 0.06 & $1 \cdot 27$ & 0.04 & 0.04 \\
\hline White rice & $-1 \cdot 11$ & 0.08 & 0.08 & $-\ddagger$ & $-\ddagger$ & $-\ddagger$ & -1.91 & 0.15 & 0.10 \\
\hline Meat & $16 \cdot 29$ & 0.30 & 0.05 & $17 \cdot 14$ & 0.32 & 0.06 & 11.87 & 0.44 & 0.02 \\
\hline Fruit & 6.82 & 0.21 & 0.03 & $7 \cdot 20$ & 0.19 & 0.04 & 5.40 & 0.18 & 0.03 \\
\hline Soft drinks & 1.88 & 0.06 & 0.03 & 2.62 & 0.09 & 0.03 & $-\ddagger$ & $-\ddagger$ & $-\ddagger$ \\
\hline Sugar and confectioneries & $7 \cdot 19$ & 0.23 & 0.03 & $7 \cdot 50$ & 0.26 & 0.03 & $6 \cdot 19$ & 0.37 & 0.01 \\
\hline Fruit and vegetable juice & 1.99 & 0.09 & 0.01 & $-\ddagger$ & $-\ddagger$ & $-\ddagger$ & $-\ddagger$ & $-\ddagger$ & $-\ddagger$ \\
\hline Alcoholic beverages & $-\ddagger$ & $-\ddagger$ & $-\ddagger$ & $5 \cdot 34$ & 0.05 & 0.27 & 4.91 & 0.16 & 0.03 \\
\hline Coffee & $-\ddagger$ & $-\ddagger$ & $-\ddagger$ & $1 \cdot 23$ & 0.03 & 0.04 & $-\ddagger$ & $-\ddagger$ & $-\ddagger$ \\
\hline
\end{tabular}

$\beta$, Regression coefficient.

${ }^{*}$ Food groups listed are those contributing at least $1 \%$ of the variation of monetary diet cost based on the stepwise regression analysis with twenty food groups (white rice; other grains; noodles; bread; other grain products; pulses; potatoes; sugar and confectioneries; fat and oil; fruit; total vegetables; fruit and vegetable juice; alcoholic beverages; green and black tea; coffee; soft drinks; fish and shellfish; meat; eggs; and dairy products) in young and middle-aged women and eighteen food groups (white rice; noodles; bread; pulses; potatoes; sugar and confectioneries; fat and oil; fruit; total vegetables; fruit and vegetable juice; alcoholic beverages; green and black tea; coffee; soft drinks; fish and shellfish; meat; eggs; and dairy products) in older women as explanatory variables and monetary diet cost as the response variable.

† Model with listed variables as explanatory variables and monetary diet cost as the response variable; regression coefficients can be interpreted as the change of monetary diet cost (Japanese yen $/ 4184 \mathrm{~kJ}$ ) with a $10 \mathrm{~g}$ increase in intake of each food group (per $4184 \mathrm{~kJ}$ ). 1 Japanese yen $=0.0099$ US dollars $=0.0088$ Euros $=0.0074$ British pounds (September 2016).

$\ddagger$ Not contributing at least $1 \%$ of the variation of monetary diet cost.

women. Supplement users had a higher mean value of monetary diet cost in young and older women but a lower value in middle-aged women. Medication users had a higher mean value of monetary diet cost in young women but a lower value in older women. Physical activity was positively associated with monetary diet cost in young and older women. For dietary reporting status, over-reporters had a higher monetary diet cost compared with under- and plausible reporters (except for middle-aged women). Age was positively associated with monetary diet cost in only middle-aged women (data not shown).

\section{Associations between monetary diet cost and nutrient intakes}

Associations of monetary diet cost with nutrient intakes are shown in Table 4. After adjustment for potential confounding 
Table 4. Associations of monetary diet cost (Japanese yen $/ 4184 \mathrm{~kJ}$ ) with nutrient intakes in young, middle-aged and older Japanese women ${ }^{\star}$ (Regression coefficients with their standard errors)

\begin{tabular}{|c|c|c|c|c|c|c|c|c|c|}
\hline & \multicolumn{3}{|c|}{ Young (n 3963) } & \multicolumn{3}{|c|}{ Middle-aged ( $n$ 3800) } & \multicolumn{3}{|c|}{ Older ( $n$ 2211) } \\
\hline & $\beta \dagger$ & SE† & Standardised $\beta$ & $\beta \dagger$ & SE† & Standardised $\beta$ & $\beta \dagger$ & SE† & Standardised $\beta$ \\
\hline Protein (\% of energy) & 1.31 & 0.03 & 0.59 & 0.94 & 0.03 & 0.48 & $2 \cdot 32$ & 0.04 & 0.76 \\
\hline Total fat ( $\%$ of energy) & 2.04 & $0 \cdot 10$ & 0.32 & 0.82 & 0.10 & 0.14 & 1.80 & 0.10 & 0.37 \\
\hline Saturated fat (\% of energy) & 0.55 & 0.04 & 0.24 & $0 \cdot 18$ & 0.03 & 0.09 & 0.35 & 0.03 & 0.23 \\
\hline Monounsaturated fat (\% of energy) & 0.85 & 0.04 & 0.31 & 0.39 & 0.04 & 0.16 & 0.57 & 0.04 & 0.30 \\
\hline Polyunsaturated fat (\% of energy) & 0.58 & 0.03 & 0.34 & 0.32 & 0.02 & 0.21 & 0.47 & 0.03 & 0.35 \\
\hline Carbohydrate (\% of energy) & -2.95 & 0.11 & -0.39 & $-2 \cdot 77$ & 0.10 & -0.39 & -3.99 & 0.12 & -0.57 \\
\hline Alcohol (\% of energy) & 0.04 & 0.01 & 0.06 & 1.32 & 0.06 & 0.32 & 0.23 & 0.04 & 0.10 \\
\hline Dietary fibre $(\mathrm{g} / 4184 \mathrm{~kJ})$ & $1 \cdot 14$ & 0.03 & 0.56 & 0.87 & 0.03 & 0.43 & $1 \cdot 12$ & 0.04 & 0.55 \\
\hline Cholesterol (mg/4184 kJ) & $16 \cdot 02$ & 1.26 & 0.21 & 9.66 & 0.93 & 0.17 & 35.82 & 1.37 & 0.50 \\
\hline $\mathrm{Na}(\mathrm{mg} / 4184 \mathrm{~kJ})$ & $235 \cdot 6$ & $10 \cdot 3$ & 0.36 & $198 \cdot 2$ & 9.9 & 0.32 & 293.6 & 8.7 & 0.59 \\
\hline $\mathrm{K}(\mathrm{mg} / 4184 \mathrm{~kJ})$ & $219 \cdot 3$ & $3 \cdot 2$ & 0.76 & $184 \cdot 0$ & 3.4 & 0.67 & $287 \cdot 3$ & $5 \cdot 3$ & 0.76 \\
\hline $\mathrm{Ca}(\mathrm{mg} / 4184 \mathrm{~kJ})$ & $37 \cdot 2$ & 1.48 & 0.39 & $27 \cdot 2$ & 1.5 & 0.30 & $67 \cdot 1$ & $1 \cdot 8$ & 0.63 \\
\hline $\mathrm{Mg}(\mathrm{mg} / 4184 \mathrm{~kJ})$ & $17 \cdot 2$ & 0.38 & 0.61 & $16 \cdot 3$ & 0.4 & 0.60 & 24.9 & 0.4 & 0.79 \\
\hline $\mathrm{Fe}(\mathrm{mg} / 4184 \mathrm{~kJ})$ & 0.64 & 0.01 & 0.68 & 0.43 & 0.01 & 0.51 & 0.83 & 0.02 & 0.75 \\
\hline Vitamin $\mathrm{A}(\mu \mathrm{g} / 4184 \mathrm{~kJ}) \ddagger$ & 84.5 & $3 \cdot 2$ & 0.40 & 57.9 & 3.0 & 0.31 & $96 \cdot 4$ & 5.0 & 0.39 \\
\hline Vitamin E $(\mathrm{mg} / 4184 \mathrm{~kJ}) \S$ & 0.74 & 0.02 & 0.62 & 0.46 & 0.02 & 0.44 & 0.70 & 0.02 & 0.71 \\
\hline Folate $(\mu \mathrm{g} / 4184 \mathrm{~kJ})$ & $40 \cdot 8$ & 0.6 & 0.76 & $31 \cdot 7$ & 0.6 & 0.66 & $46 \cdot 1$ & $1 \cdot 2$ & 0.65 \\
\hline Vitamin C (mg/4184 kJ) & $16 \cdot 3$ & 0.3 & 0.68 & $12 \cdot 4$ & 0.3 & 0.54 & 18.4 & 0.5 & 0.62 \\
\hline
\end{tabular}

$\beta$, Regression coefficient.

* Adjustment was made for survey year, residential block, size of residential area, weight status, current smoking, current alcohol drinking, dietary supplement use, medication use, physical activity and dietary reporting status. For young women, further adjustment was made for living status and eating out. For middle-aged women, further adjustment was made for eating out, occupation, education, current marital status and age. For elderly women, further adjustment was made for living status, education, current marital status and age.

† Regression coefficients can be interpreted as the change in nutrient intakes with 100 Japanese yen increase in monetary diet cost (per $4184 \mathrm{~kJ}$ ). 1 Japanese yen $=0.0099$ US dollars $=0.0088$ Euros $=0.0074$ British pounds (September 2016). All values are statistically significant $(P=0.0001$ for alcohol in young women and $P<0.0001$ for all others). $\ddagger$ Retinol equivalents.

$\S \alpha$-Tocopherol.

factors, monetary diet cost was negatively associated with total carbohydrate intake in all generations. Conversely, monetary diet cost was positively associated with intakes of all other nutrients examined, namely protein, total, saturated, monounsaturated and polyunsaturated fats, dietary fibre, $\mathrm{Na}, \mathrm{K}, \mathrm{Ca}$, $\mathrm{Mg}, \mathrm{Fe}$, vitamins $\mathrm{A}, \mathrm{E}$ and $\mathrm{C}$, and folate as well as cholesterol and alcohol intakes.

\section{Associations between monetary diet cost and food group intakes}

Table 5 presents associations between monetary diet cost and food group intakes with adjustment for potential confounding factors. Monetary diet cost was inversely associated with intakes of white rice, noodles (except for middle-aged women), bread, other grain products (not available for older women) and dairy products (only middle-aged women). There was an inverse association between monetary diet cost and sugar and confectionery intake in middle-aged and older women, with there being a positive association in young women. Monetary diet cost was also positively associated with intakes of all other food groups examined, including pulses, potatoes, fat and oil (except for middle-aged women), fruit, total vegetables, alcoholic beverages (only middle-aged women), fruit and vegetable juice, green and black tea, coffee (except for older women), soft drinks (except for older women), fish and shellfish, meat, and eggs (except for middle-aged women). The same associations between monetary diet cost and intakes of food groups (except for no associations with eggs in young women and with sugar and confectioneries, fat and oil, and eggs in older women) and nutrients were observed after excluding under- and overreporters of EI ( $n 1001$ in young women, $n 715$ in middle-aged women, and $n 662$ in older women) (data not shown).

\section{Discussion}

\section{Main findings}

To our knowledge, this is the first comprehensive study of monetary diet cost in relation to food and nutrient intakes in middle-aged and older Japanese women. Consistent with findings from Japanese female dietetic students and pregnant women $^{(27-29)}$, we found that monetary diet cost was positively associated with not only healthy dietary components (such as fruits, vegetables, fish and shellfish, dietary fibre, and key vitamins and minerals), but also less healthy components (such as saturated fat and $\mathrm{Na}$ ) across all generations of Japanese women. The major contributors to monetary diet cost were meat, total vegetables, sugar and confectioneries, fish and shellfish, white rice, and green and black tea, which are generally consistent with previous Japanese studies ${ }^{(27-29,39)}$. These foods were also major contributors to the inter-individual variation in monetary diet cost, which has not been investigated in previous studies.

\section{Comparison with previous studies}

Previous studies from Western countries have consistently shown the positive association between monetary diet cost and measures of diet quality such as energy density ${ }^{(13)}$ and 
Table 5. Associations of monetary diet cost (Japanese yen/4184 kJ) with intakes of food groups in young, middle-aged and older Japanese women* (Regression coefficients with their standard errors)

\begin{tabular}{|c|c|c|c|c|c|c|c|c|c|c|c|c|}
\hline & \multicolumn{4}{|c|}{ Young (n 3963) } & \multicolumn{4}{|c|}{ Middle-aged ( $n$ 3800) } & \multicolumn{4}{|c|}{ Older (n 2211) } \\
\hline & $\beta \dagger$ & SE† & $P$ & Standardised $\beta$ & $\beta \dagger$ & SE† & $P$ & Standardised $\beta$ & $\beta \dagger$ & SE† & $P$ & Standardised $\beta$ \\
\hline White rice & -32.9 & 1.2 & $<0.0001$ & -0.40 & $-25 \cdot 2$ & $1 \cdot 1$ & $<0.0001$ & -0.35 & -35.7 & $1 \cdot 1$ & $<0.0001$ & -0.54 \\
\hline Other grains $\ddagger \S$ & $-\|$ & $-\|$ & $-\|$ & $-\|$ & $-\|$ & $-\|$ & $-\|$ & $-\|$ & - & - & - & - \\
\hline Noodles & $-2 \cdot 7$ & 0.6 & $<0.0001$ & -0.08 & -0.6 & 0.5 & 0.19 & -0.02 & -1.5 & 0.5 & 0.002 & -0.07 \\
\hline Bread & $-4 \cdot 1$ & 0.3 & $<0.0001$ & -0.24 & $-4 \cdot 1$ & 0.3 & $<0.0001$ & -0.25 & $-3 \cdot 3$ & 0.3 & $<0.0001$ & -0.21 \\
\hline Other grain products§q & -0.7 & 0.1 & $<0.0001$ & -0.08 & -0.6 & 0.1 & $<0.0001$ & -0.08 & - & - & - & - \\
\hline Pulses & 7.4 & 0.5 & $<0.0001$ & 0.24 & 4.5 & 0.5 & $<0.0001$ & 0.16 & $6 \cdot 8$ & 0.5 & $<0.0001$ & 0.27 \\
\hline Potatoes & 3.2 & 0.2 & $<0.0001$ & 0.26 & $2 \cdot 7$ & 0.2 & $<0.0001$ & 0.22 & $4 \cdot 2$ & 0.5 & $<0.0001$ & 0.18 \\
\hline Sugar and confectioneries & 1.9 & 0.5 & $<0.0001$ & 0.07 & $-1 \cdot 8$ & 0.4 & $<0.0001$ & -0.08 & -1.7 & 0.5 & 0.001 & -0.07 \\
\hline Fat and oil & 0.8 & 0.1 & $<0.0001$ & 0.11 & 0.1 & 0.1 & 0.23 & 0.02 & 0.2 & 0.1 & 0.02 & 0.05 \\
\hline Fruit & 9.5 & 0.4 & $<0.0001$ & 0.35 & 7.7 & 0.5 & $<0.0001$ & 0.25 & $14 \cdot 1$ & 0.8 & $<0.0001$ & 0.34 \\
\hline Total vegetables & 43.9 & 0.9 & $<0.0001$ & 0.65 & 34.3 & 0.9 & $<0.0001$ & 0.55 & 43.9 & 1.4 & $<0.0001$ & 0.57 \\
\hline Alcoholic beverages & $-\|$ & $-\|$ & $-\|$ & $-\|$ & $45 \cdot 3$ & 1.5 & $<0.0001$ & 0.41 & $-\|$ & $-\|$ & $-\|$ & $-\|$ \\
\hline Fruit and vegetable juice & 8.6 & 1.0 & $<0.0001$ & 0.14 & $4 \cdot 1$ & 0.6 & $<0.0001$ & 0.11 & 3.9 & 0.9 & $<0.0001$ & 0.10 \\
\hline Green and black tea & 118.9 & 4.4 & $<0.0001$ & 0.41 & $75 \cdot 8$ & 3.8 & $<0.0001$ & 0.32 & 36.9 & 3.5 & $<0.0001$ & 0.22 \\
\hline Coffee & $10 \cdot 7$ & $1 \cdot 2$ & $<0.0001$ & 0.15 & 29.7 & 2.6 & $<0.0001$ & 0.18 & 0.0 & 1.8 & 0.99 & -0.0003 \\
\hline Soft drinks & $6 \cdot 6$ & 1.5 & $<0.0001$ & 0.07 & $5 \cdot 8$ & 1.0 & $<0.0001$ & 0.09 & 1.7 & $1 \cdot 1$ & 0.12 & 0.03 \\
\hline Fish and shellfish & 8.6 & 0.2 & $<0.0001$ & 0.52 & 7.0 & 0.2 & $<0.0001$ & 0.44 & 20.4 & 0.5 & $<0.0001$ & 0.69 \\
\hline Meat & $7 \cdot 1$ & 0.3 & $<0.0001$ & 0.36 & 4.9 & 0.3 & $<0.0001$ & 0.27 & 4.2 & 0.4 & $<0.0001$ & 0.24 \\
\hline Eggs & 0.7 & 0.3 & 0.02 & 0.04 & 0.3 & 0.2 & 0.16 & 0.02 & 0.8 & 0.3 & 0.003 & 0.07 \\
\hline Dairy products & 0.2 & $1 \cdot 2$ & 0.89 & 0.00 & -1.9 & $1 \cdot 1$ & $<0.0001$ & -0.03 & 1.2 & $1 \cdot 2$ & 0.28 & 0.02 \\
\hline
\end{tabular}

$\beta$, Regression coefficient.

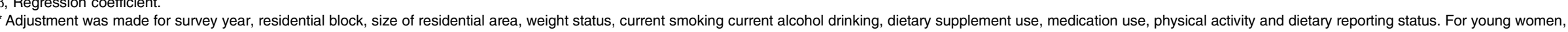

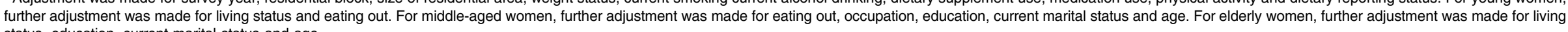
status, education, current marital status and age.

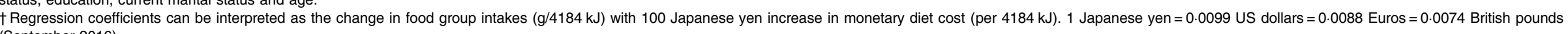
(September 2016).

¥ Including pizza, Japanese-style pancakes and cornflakes.

$\S$ Not available for older women because the brief-type diet history questionnaire used for this age group did not include these food groups.

Not examined because more than $75 \%$ or the subjects were non-consumers. 
the Healthy Eating Index ${ }^{(3,4,16,18)}$. In a representative sample of US adults, higher monetary diet cost was associated with higher consumption of vegetables, fruits, whole grains and seafood and lower consumption of refined grains, solid fat, alcohol and added sugars ${ }^{(16,18)}$. A study in young and elderly people in the Netherlands also showed that those with higher energy-dense diets consumed less of fruits and vegetables and had a lower diet $\operatorname{cost}^{(13)}$. Relatively similar associations between monetary diet cost and dietary intakes have been observed with $^{(12,15-17,19,22)}$ and without ${ }^{(6,10,13,14,21)}$ adjustment for EI, with a few exceptions ${ }^{(46)}$. These findings from Western countries are somewhat at odds with the present and previous Japanese studies ${ }^{(27-29)}$. In this study, while several 'healthy' foods (such as fruits, vegetables, and fish and shellfish) were major contributors to and positively associated with monetary diet cost, not only foods considered 'unhealthy' (such as sugar and confectionaries) but also several beverages (tea, coffee, juice and alcoholic beverages) showed similar associations with monetary diet cost. Thus, it appears that Japanese women spend more money not only on expensive but healthy foods (such as fruits, vegetables, and fish and shellfish) but also on foods consumed for pleasure or discretionary foods (such as sugar and confectionaries and beverages). This may, at least partly, explain the favourable and unfavourable characteristics of diet with higher monetary diet cost.

Only staple foods (foods with high carbohydrate contents), particularly white rice, were inversely associated with monetary diet cost. For Japanese people, white rice is a major staple food which is consumed at almost every meal, accompanied by a main and several side dishes consisting mainly of fish and shellfish, meat, eggs, vegetables and pulses. Nevertheless, white rice is inexpensive compared with the component foods of main and side dishes in Japan (which may explain the relatively small contribution of white rice to monetary diet cost). It is also probable that persons with limited money available for foods consume mainly white rice and have limited variety with respect to main and side dishes, while the reverse may be true for those with more income to spend on food. This hypothesis has some support in the observed increasing consumption of other foods such as meat, vegetables, fish and shellfish, and pulses with the increase in the Gross National Product of Japan from 1955 to $1998^{(47)}$. In addition, the National Health and Nutrition Survey in Japan has reported that lower household income is associated with higher intake of grains and lower intake of vegetables and meat ${ }^{(48)}$.

Across the generations we found that monetary diet cost was positively associated not only with intakes of nutrients such as dietary fibre and key vitamins and minerals, but also with intakes of nutrients such as saturated fat and $\mathrm{Na}$ and cholesterol and alcohol intakes, which is consistent with previous Japanese studies ${ }^{(27,29)}$. Conversely, Western studies have consistently shown that higher diet cost is associated with higher diet quality ${ }^{(6,15)}$. For example, a French study showed that higher monetary diet cost was associated with higher intakes of vitamins $\mathrm{C}, \mathrm{D}$ and $\mathrm{E}, \beta$-carotene, folates and $\mathrm{Fe}^{(6)}$. A study in low-income women in California also showed that higher monetary diet cost was related to lower intakes of saturated fat and higher intakes of vitamins $A$ and $C^{(15)}$. The higher fat (and thus lower carbohydrate) intake with increasing monetary diet cost observed here seemed to be due largely to decreasing consumption of white rice; in support of this, white rice intake was negatively correlated with the intake of total and saturated fats and cholesterol (Pearson correlation coefficient -0.17 to -0.70$)$ and positively with carbohydrate intake (0.49 to 0.65$)$. The higher intake of $\mathrm{Na}$ with increasing monetary diet cost observed in this study might be due to higher intakes of vegetables, meat, and fish and shellfish, because in Japan these foods are usually accompanied by seasonings with salty taste, such as salt, soya sauce, miso (fermented soyabean paste) and dressings. Actually, intakes of these foods were positively correlated with $\mathrm{Na}$ intake in the present study (Pearson correlation coefficient 0.08 to 0.61 ). The positive but unfavourable association between monetary diet cost and $\mathrm{Na}$ intake has also been observed in the USA ${ }^{(16-18)}$ and Spain $^{(10)}$ (not reported from other countries). Thus, reducing $\mathrm{Na}$ intake while maintaining a healthy diet may be challenging not only in Japan but also in other affluent societies.

Despite the large difference in the association of monetary diet cost with dietary intake between Japan and Western countries, positive associations between socio-economic status and monetary diet cost have been consistently observed not only in previous Western studies ${ }^{(7,8,21-23)}$ but also in the present Japanese study, where education was positively associated with monetary diet cost in middle-aged and older women. This suggests that monetary diet cost is country specific. It would also be of interest whether the present findings are similarly observed in other Asian populations.

\section{Strengths and limitations}

The major strength of the present study is a comprehensive investigation on the associations between monetary diet cost and food and nutrient intakes in three generations of women who lived over a wide geographical range of Japan and had a variety of dietary and lifestyle pattern. However, there are also several limitations. First, all our participants were female dietetic students and their mothers and grandmothers/acquaintances, and thus not a random sample of Japanese women. Given that not all Japanese adolescents enter college or university (enrollment ratio $=57 \%)^{(49)}$, the participants in this study may therefore have a relatively high socio-economic status. Additionally, although the present survey was carried out in most institutions within 1 month after the dietetic course began to minimise the influence of dietetic education, nutritional knowledge of dietetic students and their food choices are probably different from the general population or they may be more interested in or more conscious of their diet. Furthermore, the response rate was not high enough, particularly in the older generation, which may cause self-selection bias, and thus dietary habits in the present population may be healthier than those in the general population. Thus, the present results might not be generalisable to the general Japanese population, particularly for the young generation. Additionally, because the present and previous Japanese studies ${ }^{(27-29)}$ included only women, it is unknown whether the 
associations between monetary diet cost and dietary intake observed in women are similarly observed in men.

Second, all self-report dietary assessment methods are subject to both random and systematic measurement errors. To minimise them, we assessed the dietary habits during the preceding month using well-established dietary assessment questionnaires with reasonable validity in terms of commonly studied nutritional factors (DHQ and BDHQ) ${ }^{(31-33)}$, as well as the use of energy-adjusted dietary variables. Additionally, as mentioned above, exclusion of EI misreporters did not change the results of almost all analyses, which may support the robustness of the present finding.

In the absence of actual food expenditure data, food price was derived from the National Retail Price Survey and websites of nationally distributed supermarket and fast-food restaurant chains. As this procedure gives only an approximation of actual diet costs, the results of the present study should be interpreted with caution, although a similar methodology has been used in many previous studies ${ }^{(3,4,6,8,11,13)}$. Nonetheless, the satisfactory reliability of the $\mathrm{DHQ}^{(39)}$ and BDHQ (S Sasaki and $\mathrm{K}$ Murakami, unpublished results) regarding monetary diet cost against $16-\mathrm{d}$ weighed dietary record as described above may provide some assurance.

\section{Conclusion}

Contrary to Western populations, monetary diet cost was positively associated not only with healthier dietary components (such as fruits, vegetables, fish and shellfish, dietary fibre, and key vitamins and minerals) but also with less healthy components (such as saturated fat and $\mathrm{Na}$ ) in all three generations of Japanese women. The major contributors to monetary diet cost were meat, total vegetables, sugar and confectioneries, fish and shellfish, white rice, and green and black tea, which were also major contributors to the inter-individual variation in monetary diet cost. Because the relationship between food cost and dietary intake is an important health topic, further research in other populations, particularly men and children, is warranted.

\section{Supplementary material}

The supplementary material for this article can be found at https://doi.org/10.1017/jns.2017.18

\section{Acknowledgements}

The authors thank Ryoko Inomaki and Nozomi Kuriyama (Department of Nutrition, School of Human Cultures, University of Shiga Prefecture, Japan) for manuscript preparation.

The authors thank the members of the Three-Generation Study of Women on Diets and Health Study Group. The members are listed in: Kobayashi et al. ${ }^{(29)}$.

This work was supported in part by the Grants-in-Aid for Young Scientists (B) (to K. M., grant number 15K16213) from the Ministry of Education, Culture, Sports, Science and Technology of Japan and the Grants-in-Aid for Scientific
Research (A) (to S. S., grant number 22240077) from the Japan Society for the Promotion of Science. The Ministry of Education, Culture, Sports, Science and Technology of Japan and the Japan society for the Promotion of Science had no role in the design, analysis or writing of this article.

K. S. contributed to the conceptualisation of the study question, conducted the statistical analysis and prepared the first draft of the manuscript; K. M. contributed to the concept and design of the survey, conceptualised the study question, interpreted the data, provided input into the final draft of the manuscript and had primary responsibility for the final content of the manuscript; H. O. contributed to the concept and design of the survey, conceptualisation of the study question, and provided input into the final draft of the manuscript; M. B. E. L. contributed to the conceptualisation of the study question and provided input into the final draft of the manuscript; S. K., H. S. and S. S. contributed to the concept and design of the survey, coordination of the fieldwork, data collection and management, and provided input into the final draft of the manuscript. All authors read and approved the final manuscript.

There were no conflicts of interest.

\section{References}

1. Glanz K, Basil M, Maibach E, et al. (1998) Why Americans eat what they do: taste, nutrition, cost, convenience, and weight control concerns as influences on food consumption. J Am Diet Assoc 98, $1118-1126$.

2. Lennernas M, Fjellstrom C, Becker W, et al. (1997) Influences on food choice perceived to be important by nationally-representative samples of adults in the European Union. Eur J Clin Nutr 51, S8-S15.

3. Schroder H, Marrugat J \& Covas MI (2006) High monetary costs of dietary patterns associated with lower body mass index: a population-based study. Int J Obes (Lond) 30, 1574-1579.

4. Cade J, Upmeier H, Calvert C, et al. (1999) Costs of a healthy diet: analysis from the UK Women's Cohort Study. Public Health Nutr 2, 505-512.

5. Bernstein AM, Bloom DE, Rosner BA, et al. (2010) Relation of food cost to healthfulness of diet among US women. Am J Clin Nutr 92, 1197-1203.

6. Andrieu E, Darmon N \& Drewnowski A (2006) Low-cost diets: more energy, fewer nutrients. Eur J Clin Nutr 60, 434-436.

7. Darmon N \& Drewnowski A (2015) Contribution of food prices and diet cost to socioeconomic disparities in diet quality and health: a systematic review and analysis. Nutr Rev 73, 643-660.

8. Timmins KA, Hulme C \& Cade JE (2013) The monetary value of diets consumed by British adults: an exploration into sociodemographic differences in individual-level diet costs. Public Health Nutr 18, 151-159.

9. Morris MA, Hulme C, Clarke GP, et al. (2014) What is the cost of a healthy diet? Using diet data from the UK Women's Cohort Study. J Epidemiol Commun Health 68, 1043-1049.

10. Lopez CN, Martinez-Gonzalez MA, Alonso A, et al. (2009) Cost of compliance with daily recommended values of micronutrients among a cohort of Spanish university graduates: the SUN (Seguimiento Universidad de Navarra) Study. Public Health Nutr 12, 2092-2096.

11. Maillot M, Darmon N, Vieux F, et al. (2007) Low energy density and high nutritional quality are each associated with higher diet costs in French adults. Am J Clin Nutr 86, 690-696.

12. Marty L, Dubois C, Gaubard MS, et al. (2015) Higher nutritional quality at no additional cost among low-income households: insights from food purchases of "positive deviants". Am J Clin Nutr 102, 190-198. 
13. Waterlander WE, de Haas WE, van Amstel I, et al. (2010) Energy density, energy costs and income - how are they related? Public Health Nutr 13, 1599-1608.

14. Townsend MS, Aaron GJ, Monsivais P, et al. (2009) Less-energy-dense diets of low income women in California are associated with higher energy-adjusted diet costs. Am J Clin Nutr 89, 1220-1226.

15. Rehm CD, Monsivais P \& Drewnowski A (2011) The quality and monetary value of diets consumed by adults in the United States. Am J Clin Nutr 94, 1333-1339.

16. Drewnowski A, Rehm CD, Maillot M, et al. (2014) The relation of potassium and sodium intakes to diet cost among US adults. I Hum Hypertens 29, 14-21.

17. Rehm CD, Monsivais P \& Drewnowski A (2015) Relation between diet cost and Healthy Eating Index 2010 scores among adults in the United States 2007-2010. Prev Med 73, 70-75.

18. Darmon N, Darmon M, Maillot M, et al. (2005) A nutrient density standard for vegetables and fruits: nutrients per calorie and nutrients per unit cost. J Am Diet Assoc 105, 1881-1887.

19. Ledikwe JH, Blanck HM, Khan LK, et al. (2006) Low-energy-density diets are associated with high diet quality in adults in the United States. J Am Diet Assoc 106, 1172-1180.

20. Aggarwal A, Monsivais P, Cook AJ, et al. (2011) Does diet cost mediate the relation between socioeconomic position and diet quality? Eur J Clin Nutr 65, 1059-1066.

21. Monsivais P \& Drewnowski A (2009) Lower-energy-density diets are associated with higher monetary costs per kilocalorie and are consumed by women of higher socioeconomic status. I Am Diet Assoc 109, 814-822.

22. Ryden PJ \& Hagfors L (2011) Diet cost, diet quality and socioeconomic position: how are they related and what contributes to differences in diet costs? Public Health Nutr 14, 1680-1692.

23. Darmon N \& Drewnowski A (2008) Does social class predict diet quality? Am J Clin Nutr 87, 1107-1117.

24. Mayen AL, Marques-Vidal P, Paccaud F, et al. (2014) Socioeconomic determinants of dietary patterns in low- and middle-income countries: a systematic review. Am J Clin Nutr 100, 1520-1531.

25. Honjo K, Tsutsumi A, Kayaba K, et al. (2010) Socioeconomic indicators and cardiovascular disease incidence among Japanese community residents: the Jichi Medical School Cohort Study. Int J Behav Med 17, 58-66.

26. Martikainen P, Ishizaki M, Marmot MG, et al. (2001) Socioeconomic differences in behavioural and biological risk factors: a comparison of a Japanese and an English cohort of employed men. Int J Epidemiol 30, 833-838.

27. Murakami K, Sasaki S, Okubo H, et al. (2007) Monetary costs of dietary energy reported by young Japanese women: association with food and nutrient intake and body mass index. Public Health Nutr 10, 1430-1439.

28. Murakami K, Sasaki S, Takahashi Y, et al. (2009) Monetary cost of self-reported diet in relation to biomarker-based estimates of nutrient intake in young Japanese women. Public Health Nutr 12, 1290-1297.

29. Murakami K, Miyake Y, Sasaki S, et al. (2009) Monetary diet cost is associated with not only favorable but also unfavorable aspects of diet in pregnant Japanese women: the Osaka Maternal and Child Health Study. Environ Health Insights 3, 27-35.

30. Kobayashi S, Asakura K, Suga H, et al. (2013) High protein intake is associated with low prevalence of frailty among old Japanese women: a multicenter cross-sectional study. Nutr J 12, 164.

31. Kobayashi S, Murakami K, Sasaki S, et al. (2011) Comparison of relative validity for food group intake estimated by comprehensive and brief-type self-administered diet history questionnaires against 16 d dietary records in Japanese adults. Public Health Nutr 14, $1200-1211$.
32. Kobayashi S, Honda S, Murakami K, et al. (2012) Both comprehensive and brief self-administered diet history questionnaires satisfactorily rank nutrient intakes in Japanese adults. J Epidemiol 22, 151-159.

33. Sasaki S, Yanagibori R \& Amano K (1998) Self-administered diet history questionnaire developed for health education: a relative validation of the test-version by comparison with 3-day diet record in women. J Epidemiol 8, 203-215.

34. Sasaki S, Ushio F \& Amano K (2000) Serum biomarker-based validation of a self-administered diet history questionnaire for Japanese subjects. J Nutr Sci Vitaminol 46, 285-296.

35. Science and Technology Agency (2005) Standard Tables of Food Composition in Japan, fifth revised and enlarged edition. Tokyo, Japan: Printing Bureau of the Ministry of Finance (in Japanese).

36. Livingstone MBE \& Black AE (2003) Markers of the validity of reported energy intake. J Nutr 133, 895S-920S.

37. Murakami K, Sasaki S, Uenishi K, et al. (2012) The degree of misreporting of the energy-adjusted intake of protein, potassium, and sodium does not differ among under-, acceptable, and overreporters of energy intake. Nutr Res 32, 741-750.

38. Price Statistic Office, Statistic Bureau (2004) National Retail Price Survey in Japan. http://www.stat.go.jp/data/kouri/index. $\mathrm{htm}$ (accessed January 2016) (in Japanese).

39. Murakami K, Sasaki S, Takahashi Y, et al. (2008) Comparability of weighed dietary records and a self-administered diet history questionnaire for estimating monetary cost of dietary energy. Environ Health Insights 1, 35-43.

40. World Health Organization (2000) Obesity: Preventing and Managing the Global Epidemic. Report of a WHO Consultation. WHO Technical Report Series no. 894. Geneva: WHO.

41. WHO Expert Consultation (2004) Appropriate body-mass index for Asian populations and its implications for policy and intervention strategies. Lancet 363, 157-163.

42. Ainsworth BE, Haskell WL, Herrmann SD, et al. (2011) Compendium of Physical Activities: a second update of codes and MET values. Med Sci Sports Exerc 43, 1575-1581.

43. Black AE (2000) Critical evaluation of energy intake using the Goldberg cut-off for energy intake:basal metabolic rate. A practical guide to its calculation, use and limitations. Int J Obes Relat Metab Disord 24, 1119-1130.

44. Ganpule AA, Tanaka S, Ishikawa-Takata K, et al. (2007) Interindividual variability in sleeping metabolic rate in Japanese subjects. Eur J Clin Nutr 61, 1256-1261.

45. Miyake R, Tanaka S, Ohkawara K, et al. (2011) Validity of predictive equations for basal metabolic rate in Japanese adults. J Nutr Sci Vitaminol 57, 224-232.

46. Rao M, Afshin A, Singh G, et al. (2013) Do healthier foods and diet patterns cost more than less healthy options? A systematic review and meta-analysis. BMJ Open 3, e004277.

47. Cabinet Office, Department of National Accounts (1998) National Accounts Statistics: Gross domestic product and total expenditure calculation. http://www.e-stat.go.jp/SG1/estat/GL02010201.do? method $=$ searchTop\&and Keyword $=\% \mathrm{E} 5 \% 9 \mathrm{~B} \% \mathrm{BD} \% \mathrm{E} 6 \% \mathrm{~B} 0 \% 91 \%$ $\mathrm{E} 7 \% \mathrm{~B} 7 \% 8 \mathrm{~F} \% \mathrm{E} 7 \% 94 \% 9 \mathrm{~F} \% \mathrm{E} 7 \% 94 \% \mathrm{~A} 3$ (in Japanese) (accessed January 2016).

48. Ministry of Health, Labour and Welfare, Japan (2015) The National Health and Nutrition Survey in Japan, 2014. http://www.mhlw.go. jp/file/04-Houdouhappyou-10904750-Kenkoukyoku-Gantaisakuk enkouzoushinka/0000106547.pdf (in Japanese) (accessed January 2016).

49. Ministry of Education, Culture, Sports, Science and Technology (2014) General Survey of School, 2014. http://www.mext.go.jp/ component/b_menu/houdou/_icsFiles/afieldfile/2014/08/07/ 1350732_01.pdf (in Japanese) (accessed January 2016). 